\title{
SELF-REPORTED ORAL HEALTH BEHAVIOUR AMONG SCANDINAVIAN AND POLISH MEDICAL STUDENTS STUDYING IN POLAND
}

\author{
Ewa Rodakowska', Anna Kierklo², Jacek Jamiołkowski ${ }^{3}$ \\ ${ }^{1}$ Department of Restorative Dentistry, Medical University of Bialystok, Bialystok, Poland \\ ${ }^{2}$ Department of Dentistry Propaedeutics, Medical University of Bialystok, Bialystok, Poland \\ ${ }^{3}$ Department of Public Health, Medical University of Bialystok, Bialystok, Poland
}

\section{SUMMARY}

Aim: The aim of the study was to assess the self-reported oral health behaviour of Scandinavian and Polish medical students studying in Poland.

Methods: Three hundred and fifty subjects took part in the questionnaire survey. They were the 4th to 6 th year Scandinavian and Polish medical students attending the same medical school. Test-retest reliability demonstrated an accepted level of kappa $>0.50$. The questionnaire comprised issues dealing with demographics, dental health behaviour, fluoride tablet recommendation, self-rated oral health, and smoking habit. Statistical analyses were performed using the Fisher-Freeman-Halton test, Fisher's exact test and the multiple logistic regression. A p value $<0.05$ was considered significant.

Results: Gender and nationality statistical differences were observed regarding the frequency of visiting a dentist: more Polish females visited a dentist less than a year ago $(p=0.011)$, the reasons for visiting a dentist - check up, filling $(p=0.002 ; p=0.040)$; the frequency of tooth brushing was higher among Polish females compared to Polish males and Scandinavian females $(p<0.001 ; p<0.001)$. Polish males ate sweet snacks significantly more often than Polish females and Scandinavian males $(p=0.018 ; p=0.004)$. In the logistic regression analysis factors were independently associated with visiting a dentist at least once a year: female gender $(\mathrm{OR}=2.310 ; 95 \% \mathrm{Cl} 1.381-3.865)$, Polish nationality $(\mathrm{OR}=3.833$; $95 \% \mathrm{Cl} 2.293-6.408)$. Associations with the female gender were significant for the following dependent variables: visiting a dentist more than a year ago ( $O R=1.913 ; 95 \% \mathrm{Cl} 1.192-3.070)$, brushing teeth at least once a day $(\mathrm{OR}=3.759 ; 95 \% \mathrm{Cl} 1.567-9.017)$, and use of dental floss $(\mathrm{OR}=2.249 ; 95 \% \mathrm{Cl} 1.445-3.503)$. Polish nationality was associated with an increasing rate of brushing teeth for at least 3 minutes $(\mathrm{OR}=2.435$; $95 \% \mathrm{Cl} 1.526-3.885)$, and smoking cigarettes ( $\mathrm{OR}=2.340$; $95 \% \mathrm{Cl} 1.336-4.098)$.

Conclusions: Better prognosis for maintaining good oral health was established in the Scandinavian group of students. Polish females took greater care of their teeth than Polish males and the majority of their Scandinavian counterparts.

Key words: medical students, oral health behaviour, questionnaire, smoking, dental education

Address for correspondence: E. Rodakowska, Department of Restorative Dentistry, Medical University of Bialystok, UI. Sklodowskiej 24, 15-274 Bialystok, Poland. E-mail: ewarodakowska@interia.pl

http://dx.doi.org/10.21101/cejph.a4084

\section{INTRODUCTION}

Oral health is strongly determined by socio-cultural norms and the dental health system which may differ among countries. Behavioural risk factors that act directly or almost directly are oral hygiene practices, one's individual experience, dietary habits (sugar consumption), tobacco use, and excessive consumption of alcohol $(1,2)$. The most common pathology in the oral cavity is dental caries. It is a deterioration of hard tissues of teeth that affects almost everybody during the lifespan. It may cause discomfort and problems in both private and public life. They include difficulties with eating, chewing, speaking and smiling (missed, damaged, discoloured teeth), toothaches or school and work absence. Further general consequences of poor oral health including periodontal diseases are diabetes mellitus, cardiovascular diseases or pregnancy complications (3).

In industrialized countries there has been considerable improvement in the dental health in adults in the past three decades.
This applies to Scandinavian countries where a decrease in dental caries is well documented (4). In Norwegian adults, there was an $83 \%$ reduction in caries prevalence from 1973-2003. The proportion of individuals with no detected dental caries gradually increased from 5\% in 1973 to $54 \%$ in 2003 (5). On the other hand there is hardly any available information in literature about the prevalence of dental caries in Poland where it is still an ongoing problem among both young and adults. For instance in 2010, a nationwide oral health monitoring revealed that $82.7 \%$ of 12 year old children and $99.9 \%$ of $35-44$ year old adults had dental caries (6). These data are appalling.

In Poland, the Ministry of Science and Higher Education defined the minimum dental education requirements for medical students. A dental course for medical students is mandatory and should last at least 15 hours in the 4th year of the 6-year course. However, it seems to be limited to the proper exposure of problems concerning oral health. Generally dental issues are left to be dealt with solely by oral health experts (7). Loster and Likeman (8) 
believe that a basic knowledge of dentistry in medical students enables better communication between medical professionals and dentists. It is obvious that medical practitioners will encounter patients manifesting oral and dental problems in the future. Oral health is an essential component of human health and integrated part of health related quality of life $(9,10)$. Also, regardless of university education, students have their own experience of visiting the dentists and present certain attitudes towards oral health stemming from their families, culture, experience, lifestyle, beliefs, and/or finances (11). Therefore, medical students even if they come from different countries will take part in shaping healthrelated behaviour, promoting health and preventing diseases in their respective environments $(12,13)$.

The aim of the study was to assess self-reported oral health behaviour of Scandinavian and Polish medical students studying in Poland.

\section{MATERIALS AND METHODS}

\section{Study Population}

The subjects of the survey were recruited by means of the convenience sample. They were the 4 th, 5 th and 6 th year Scandinavian and Polish medical students attending the Medical University of Bialystok, Poland. A total number of 490 individuals responded to the questionnaire; $102(20.9 \%)$ did not complete questionnaire fully and were rejected. The estimated sample of subjects was 350,122 Scandinavian (65 males and 57 females) and 228 Polish ( 82 males and 146 females). The response rate and distribution of students by academic year are shown in Table 1. Scandinavian students declared to be of various nationalities: Norwegian (76), Swedish (39) and 7 others. Polish students' average age was 23.44-23.32 in females and 23.64 in males. Scandinavian students' average age was 25.05-25.07 in females and 25.03 in males.

\section{Data Collection}

The research was approved by the Ethics Committee of the Medical University of Bialystok. The self-prepared 22 items questionnaire was written in Polish and this version was completed by the Polish students. The questionnaire was also translated into English. The translation was performed by a professional translator specializing in medicine. This English version was translated back into Polish by a bilingual dental professional who then translated it again into English. The two English translations were compared and verified for linguistic equivalence by the review committee and the final version was delivered to the Scandinavian students. However, in order to check reliability of the questionnaire, a convenience sample of 30 medical students had been asked to fill in the questionnaire twice over a period of 2 weeks prior to the main study. The kappa coefficient was used to measure test-retest reliability. The majority of items demonstrated a good level of reliability for medical students with kappa scores greater than 0.50 . The only items that demonstrated lower reproducibility were: "Do you eat snacks between meals?" (kappa =0.442); "How often do you eat sweet snacks?" (kappa $=0.420)$; and "Do you pay attention to tooth paste you buy?" (kappa $=0.474)$.
Participation in the survey was anonymous and voluntary. The students were informed of the purpose of the study and filled in the questionnaire after the dental course was finished. We communicated with Scandinavian students in English. During the completion of the questionnaire the investigator was always available. The questions were close-ended, students were asked to choose only one response. The questionnaire comprised issues dealing with demographics (gender, age, nationality), dental health behaviour (frequency and reasons of dental visits, tooth brushing, use of additional devices like dental floss, mouth wash, sugar free gum, dental sticks, tongue scrapes and interdental brushes, as well as consumption of sugar), fluoride recommendation, self-rated oral health, and smoking habit. A separate question was prepared for Scandinavian students regarding their change in dental habits while studying in Poland $-67.7 \%$ of males and $70.2 \%$ of females did not change their dental habits while studying in Poland and this difference was not statistically significant.

\section{Statistical Analyses}

For variables with more than two categories the FisherFreeman-Halton test was used. To evaluate relations between nominal variables the Fisher's exact test was used. The multiple logistic regression analysis was performed to study the association between oral health behaviour variables and selected factors (nationality and gender). Oral health behaviour variables were: visiting a dentist at least once a year and more than a year ago; brushing teeth at least once a day and for at least 3 minutes, use of electrical toothbrush; eating snacks occasionally, eating sweets less often than daily; very good opinion of own teeth; taking fluoride tablets as a child, paying attention to toothpaste, use of dental floss, mouth-wash, sugar free gum, dental sticks, tongue scrapes, and interdental brushes; and cigarettes smoking. Odds ratios (OR) and their confidence intervals (95\% CI) were estimated. Statistical analysis was performed using the statistical package IBM ${ }^{\circledR}$ SPSS ${ }^{\circledR}$ Statistics 20.0. Statistical results with a $\mathrm{p}$ value lower than 0.05 were considered significant.

\section{RESULTS}

Table 2 presents the distribution of responses to the questionnaire among Scandinavian and Polish students according to nationality and gender. There were many significant differences in oral hygiene between Scandinavian and Polish medical students and between males and females. Gender and nationality statistical differences were observed regarding the frequency of visiting a dentist: significantly more Polish females visited a dentist less than a year ago $(p=0.011)$. Nationality and gender statistical differences were observed in the reasons for visiting a dentist check up, filling ( $p=0.002 ; p=0.040)$. Rate of tooth brushing at least twice daily was significantly higher among Polish females compared to Polish males and Scandinavian females $(\mathrm{p}<0.001$; $p<0.001$ ). Also Polish females brushed their teeth significantly longer than Polish males and their Scandinavian peers $(p<0.001)$. Polish males ate sweet snacks significantly more often than Polish females and Scandinavian males $(p=0.018 ; p=0.004)$. It was also found that fluoride tablets were recommended as a part of childhood dental regime and administrated in Scandinavian countries 
contrary to Poland where only a small part of participants had used them. Statistically significant differences were observed between the two nationalities $(\mathrm{p}<0.001)$. Significant differences in the Polish group were observed in smoking habits where Polish males smoked the most (39.0\%).

Scandinavian and Polish medical students used various additional dental devices (Table 3). Polish female students statistically significantly paid attention to toothpaste, and more often used dental floss, mouth-wash, tongue scrapes and interdental brushes than Polish males. Another significant difference was observed between Polish and Scandinavian males regarding the use of sugar free gum and tongue scrapes.

In the logistic regression analysis (Table 4) two analyzed factors were independently associated with visiting a dentist at least once a year: female gender $(\mathrm{OR}=2.310 ; 95 \%$ CI $1.381-3.865)$ and Polish nationality $(\mathrm{OR}=3.833 ; 95 \%$ CI 2.293-6.408). Associations only with the female gender were significant for the following dependent variables: visiting a dentist more than a year ago (OR=1.913; 95\% CI 1.192-3.070), brushing teeth at least once a day $(\mathrm{OR}=3.759 ; 95 \% \mathrm{CI} 1.567-9.017)$, paying attention to toothpaste $(\mathrm{OR}=2.386 ; 95 \%$ CI $1.274-4.471)$, use of dental floss $(\mathrm{OR}=2.249 ; 95 \%$ CI $1.445-3.503)$, and use of interdental brushes $(\mathrm{OR}=5.341 ; 95 \%$ CI 1.199-23.782). For other variables we indicated associations only with the Polish nationality. Polish nationality was associated with an increasing rate of brushing teeth for at least 3 minutes ( $\mathrm{OR}=2.435 ; 95 \%$ CI $1.526-3.885)$, using sugar free gum $(\mathrm{OR}=1.723 ; 95 \%$ CI $1.098-2.704)$ and smoking cigarettes $(\mathrm{OR}=2.340 ; 95 \% \mathrm{CI} 1.336-4.098)$, as well as with a decreasing rate of eating sweets less often than daily $(\mathrm{OR}=0.446,95 \% \mathrm{CI} 0.283-0.703)$ and taking fluoride tablets as a child $(\mathrm{OR}=0.064 ; 95 \% \mathrm{CI} 0.036-0.113)$. Table 5 presents the distribution between four selected statements describing positive and negative oral health behaviour: dental visits at least once a year, twice daily tooth brushing, use of dental floss and eating sweets at least once a day in the 4th, 5th and 6th year of study. Significant differences were not observed in either year of the study in either nationality.

\section{DISCUSSION}

We presented a cross-cultural and gender comparison of selfrated oral health in medical students. Moreover, this is the first study in Poland in which the self-rated oral health behaviour among foreign and Polish students of medicine studying together at one Polish university was compared. No Polish data comparable to this study can be found in literature. Although many studies have been carried out regarding oral health behaviour, most of them only among dental professionals $(11,14,15)$.
Our results showed several significant differences in oral health behaviour between Scandinavian and Polish medical students and males and females. We observed a satisfactory picture of oral health behaviour of Scandinavian and Polish students and both males and females. According to Kumar et al. (16) students of health professions are more concerned with the oral hygiene than other students and people in general, and according to Paulander et al. (17) education influences oral hygiene in adults.

On the other hand the results showed some significant differences among the Polish students. Polish females had adopted very good oral hygiene in comparison to males, which is very promising in the context of the high frequency of dental caries in Poland $(6,18)$. It is difficult to explain whether they maintained better oral health behaviour only because of gender disparities or due to the professional knowledge they acquired as future health providers. Generally, it has been proven that women present better oral health behaviour than men (19-21).

Therefore, in our study oral health behaviour was determined by gender and by nationality. Dental behaviour performance of Polish female students differed statistically in their favour in almost every aspect compared to their Scandinavian counterparts. In comparison with students from other countries, Italian students (21) also demonstrated a similar high frequency of at least twice daily tooth brushing (92.1\%) alike Scandinavian students (males $86.2 \%$, females $94.9 \%$ ), but their use of dental floss was at a lower rate (33.2\%) compared to Polish students. On the other hand Turkish students visited dentists almost as often as our students (57\%), but had definitely worse oral health behaviour - only $53.7 \%$ brushed their teeth twice a day, $18.8 \%$ used mouthwash and $3 \%$ cleaned interdental spaces using dental floss (20). The majority of Kuwait male health science students brushed their teeth once a day only, which shows that a twice daily routine was not very common, but over $60 \%$ of them had visited a dentist during the last year (22). On the other hand in some parts of the world the frequency of twice daily brushing is quite low even among dental professionals, for instance in Nigeria 47.5\% (8) and Iran 57\% (19).

A lack of concern for smoking in Polish medical students is quite disturbing. They smoke significantly more (over twice as much) than Scandinavian students $(\mathrm{OR}=2.340 ; 95 \% \mathrm{CI}$ 1.336-4.098). Among Polish future health providers 39.0\% of males and almost one quarter of females smoked. Unfortunately, according to the WHO data they smoke at the same rate as the general Polish population (23), which is particularly surprising as medical university curriculum covers smoking hazards and tobacco depending diseases. Moreover, smoking can present significant risk factors for dental caries (24). The overall prevalence of smoking rate among medical students in other countries was lower and ranged from $3.3 \%$ in Canada to $19.3 \%$ in Turkey $(25,26)$. The implementation of a tobacco curriculum and smok-

Table 1. The response rate and distribution of students by the academic year

\begin{tabular}{|l|c|c|}
\hline \multirow{2}{*}{ Academic year } & \multicolumn{2}{|c|}{ Students' participation rate; $\mathbf{n}(\%)$} \\
\cline { 2 - 3 } & Scandinavian & Polish \\
\hline 4th & $26(52.0)$ & $59(34.0)$ \\
\hline th & $49(98.0)$ & $79(44.0)$ \\
\hline 6th & $47(94.0)$ & $90(49.0)$ \\
\hline Total & $122(81.3)$ & $228(42.3)$ \\
\hline
\end{tabular}


Table 2. The distribution of responses to the questionnaire among Polish and Scandinavian students, males, females (n/\%)

\begin{tabular}{|c|c|c|c|c|c|c|}
\hline \multirow{2}{*}{ Variable } & \multirow{2}{*}{ Variants } & \multicolumn{2}{|c|}{ Scandinavian } & \multicolumn{2}{|c|}{ Polish } & \multirow{2}{*}{$p$ value } \\
\hline & & Males & Females & Males & Females & \\
\hline \multirow{5}{*}{$\begin{array}{l}\text { How often do you visit } \\
\text { the dentist? }\end{array}$} & Every 3 months & $0(0.0)$ & $2(3.5)$ & $4(4.8)$ & $20(13.8)$ & 1: 0.014 \\
\hline & Every 6 months & $9(13.8)$ & $2(3.5)$ & $17(20.8)$ & $56(38.3)$ & $2:<0.001$ \\
\hline & Once a year & $22(33.9)$ & $33(58.0)$ & $41(50.0)$ & $54(37.0)$ & 3: 0.001 \\
\hline & Every 2 years & $16(24.6)$ & $8(14.0)$ & $4(4.9)$ & $9(6.1)$ & $4:<0.001$ \\
\hline & Toothache & $18(27.7)$ & $12(21.0)$ & $16(19.5)$ & $7(4.8)$ & \\
\hline \multirow{3}{*}{$\begin{array}{l}\text { When did you last see } \\
\text { a dentist? }\end{array}$} & $<1$ year ago & $38(58.4)$ & $39(68.4)$ & $53(64.8)$ & $117(80.1)$ & \\
\hline & $1-2$ years ago & $18(27.8)$ & $14(24.6)$ & $20(24.3)$ & $25(17.1)$ & 2: 0.011 \\
\hline & $\geq 3$ years ago & $9(13.8)$ & $4(7.0)$ & $9(10.9)$ & $4(2.8)$ & \\
\hline \multirow{6}{*}{$\begin{array}{l}\text { What was the reason } \\
\text { for your last visit? }\end{array}$} & Check-up & $42(64.8)$ & $32(56.1)$ & $36(44.0)$ & $72(49.3)$ & \\
\hline & Filling & $4(6.1)$ & $4(7.0)$ & $24(29.2)$ & $33(22.7)$ & 2: 0.03 \\
\hline & Extraction & $2(3.0)$ & $7(12.3)$ & $4(5.0)$ & $6(4.1)$ & 3: 0.002 \\
\hline & Toothache & $9(13.9)$ & $5(8.8)$ & $14(17.0)$ & $12(8.2)$ & $4: 0.04$ \\
\hline & Prof. cleaning & $6(9.2)$ & $5(8.8)$ & $3(3.6)$ & $9(6.2)$ & \\
\hline & Orthod. treatment & $2(3.0)$ & $4(7.0)$ & $1(1.2)$ & $14(9.5)$ & \\
\hline \multirow{4}{*}{$\begin{array}{l}\text { How often do you } \\
\text { brush your teeth? }\end{array}$} & After every meal & $4(6.1)$ & $1(1.7)$ & $11(13.4)$ & $47(32.2)$ & \\
\hline & Twice a day & $56(86.2)$ & $54(94.9)$ & $58(70.8)$ & 93 (63.7) & $2:<0.001$ \\
\hline & Once a day & $4(6.1)$ & $1(1.7)$ & $12(14.6)$ & $6(4.1)$ & $4:<0.001$ \\
\hline & $<$ once a day & $1(1.6)$ & $1(1.7)$ & $1(1.2)$ & $0(0.0)$ & \\
\hline \multirow{4}{*}{$\begin{array}{l}\text { For how long do you } \\
\text { brush your teeth? }\end{array}$} & Less than 1 minute & $3(4.6)$ & $1(1.8)$ & $4(4.9)$ & $4(2.8)$ & \\
\hline & One minute & $24(37.0)$ & $30(52.7)$ & $20(24.3)$ & $35(24.0)$ & \\
\hline & Three minutes & $34(52.3)$ & $21(36.8)$ & $51(62.2)$ & $91(62.3)$ & \\
\hline & Over 5 minutes & $4(6.1)$ & $5(8.7)$ & $7(8.6)$ & $16(10.9)$ & $4:<0.001$ \\
\hline \multirow{3}{*}{$\begin{array}{l}\text { What kind of } \\
\text { toothbrush do you } \\
\text { use? }\end{array}$} & Manual & $46(70.8)$ & $49(86.0)$ & $65(79.3)$ & $110(75.3)$ & \\
\hline & Electrical & $9(13.9)$ & $5(8.8)$ & $10(12.2)$ & $21(14.4)$ & \\
\hline & Both types & $10(15.3)$ & $3(5.2)$ & $7(8.5)$ & $15(10.3)$ & \\
\hline \multirow{3}{*}{$\begin{array}{l}\text { Do you eat sweet } \\
\text { snacks between } \\
\text { meals? }\end{array}$} & Yes & $24(37.0)$ & $23(40.4)$ & $44(53.7)$ & $64(43.9)$ & \\
\hline & No & $9(13.8)$ & $11(19.2)$ & $12(14.6)$ & $27(18.4)$ & \\
\hline & Occasionally & $32(49.2)$ & $23(40.4)$ & $26(31.7)$ & $55(37.7)$ & \\
\hline \multirow{4}{*}{$\begin{array}{l}\text { How often do you eat } \\
\text { sweet snacks? }\end{array}$} & Once a week & 34 (52.3) & $21(36.9)$ & $23(28.0)$ & $54(37.0)$ & \\
\hline & Once a day & $20(30.8)$ & $25(43.9)$ & $38(46.3)$ & $62(42.5)$ & 2: 0.018 \\
\hline & Between meals & $6(9.2)$ & $6(10.5)$ & $16(19.6)$ & $19(13.0)$ & 3: 0.004 \\
\hline & Never & $5(7.7)$ & $5(8.7)$ & $5(6.1)$ & $11(7.5)$ & \\
\hline \multirow{4}{*}{$\begin{array}{l}\text { Your opinion of the } \\
\text { health status of your } \\
\text { teeth }\end{array}$} & Very good & $22(33.9)$ & $15(26.3)$ & $16(19.5)$ & $28(19.2)$ & \\
\hline & Good & $31(47.7)$ & $29(50.9)$ & $45(54.9)$ & $81(55.5)$ & \\
\hline & Average & $10(15.3)$ & $12(21.0)$ & $19(23.2)$ & $35(24.0)$ & \\
\hline & Poor & $2(3.1)$ & $1(1.8)$ & $2(2.4)$ & $2(1.3)$ & \\
\hline \multirow{2}{*}{$\begin{array}{l}\text { Fluoride tablets } \\
\text { recommendation }\end{array}$} & Yes & $46(70.8)$ & $39(48.4)$ & $11(13.4)$ & $25(17.1)$ & $3:<0.001$ \\
\hline & No & $19(29.2)$ & $18(31.6)$ & $71(86.6)$ & $121(82.9)$ & $4:<0.001$ \\
\hline \multirow{2}{*}{$\begin{array}{l}\text { Taking fluoride tablets } \\
\text { as a child }\end{array}$} & Yes & $44(67.7)$ & $36(63.1)$ & $7(8.5)$ & $18(12.3)$ & $3:<0.001$ \\
\hline & No & $21(32.3)$ & $21(36.9)$ & $75(91.5)$ & $128(87.7)$ & $4:<0.001$ \\
\hline \multirow{3}{*}{$\begin{array}{l}\text { Do you smoke } \\
\text { cigarettes? }\end{array}$} & Yes & $1(1.5)$ & $5(8.8)$ & $32(39.0)$ & $36(24.6)$ & $2: 0.03$ \\
\hline & No & 54 (83.1) & $47(82.4)$ & $49(59.8)$ & $109(74.7)$ & $3:<0.001$ \\
\hline & Occasionally & $10(15.4)$ & $5(8.8)$ & $1(1.2)$ & $1(0.7)$ & $4:<0.001$ \\
\hline
\end{tabular}

1: Scandinavian males vs. females; 2: Polish males vs. females; 3: Scandinavian males vs. Polish; 4: Scandinavian females vs. Polish; Fisher-Freeman-Halton test; $p<0.05$ 
Table 3. The distribution of responses among Polish and Scandinavian students, males, females ( $n / \%)$

\begin{tabular}{|c|c|c|c|c|c|c|}
\hline & & \multicolumn{2}{|c|}{ Scandinavian } & \multicolumn{2}{|c|}{ Polish } & \multirow{2}{*}{$p$ value } \\
\hline & & Males & Females & Males & Females & \\
\hline \multirow{2}{*}{$\begin{array}{l}\text { Paying attention to } \\
\text { toothpaste you buy }\end{array}$} & Yes & $53(81.6)$ & $48(84.2)$ & 64 (78.1) & $72(93.2)$ & $2:<0.001$ \\
\hline & No & $12(18.4)$ & $9(15.8)$ & $18(21.9)$ & $10(6.8)$ & \\
\hline \multicolumn{7}{|c|}{ What else do you use in addition to tooth brushing? } \\
\hline \multirow{2}{*}{ Dental floss } & Yes & $34(52.3)$ & $38(66.7)$ & $33(40.2)$ & $92(63.0)$ & 2: 0.01 \\
\hline & No & $31(4776)$ & 19 (33.3) & $49(59.8)$ & $54(37.0)$ & \\
\hline \multirow{2}{*}{ Mouthwash } & Yes & $42(64.7)$ & 36 (63.1) & $45(54.9)$ & $105(72.0)$ & 2: 0.01 \\
\hline & No & $23(35.3)$ & $21(36.9)$ & $37(45.1)$ & $41(28.0)$ & \\
\hline \multirow{2}{*}{ Sugar free gum } & Yes & $24(37.0)$ & $29(50.9)$ & $45(54.9)$ & $88(60.2)$ & 3: 0.03 \\
\hline & No & $41(63.0)$ & $28(49.1)$ & $37(45.1)$ & $58(39.8)$ & \\
\hline \multirow{2}{*}{ Dental sticks } & Yes & $11(17.0)$ & $9(15.8)$ & $20(24.3)$ & $22(15.0)$ & \\
\hline & No & $54(83.0)$ & 48 (84.2) & $62(75.7)$ & $124(85.0)$ & \\
\hline \multirow{2}{*}{ Tongue scraps } & Yes & $12(18.4)$ & $11(19.2)$ & $6(7.3)$ & $28(17.1)$ & $2: 0.04$ \\
\hline & No & $53(81.6)$ & $46(80.8)$ & 76 (92.7) & $121(82.9)$ & $3: 0.04$ \\
\hline \multirow{2}{*}{ Interdental brushes } & Yes & $0(0.0)$ & $2(3.6)$ & $2(2.4)$ & $14(9.6)$ & $2: 0.05$ \\
\hline & No & $65(100.0)$ & $55(96.4)$ & $80(97.6)$ & $132(90.4)$ & \\
\hline
\end{tabular}

1: Scandinavian males vs. females; 2: Polish males vs. females; 3: Scandinavian males vs. Polish; 4: Scandinavian females vs. Polish; Fisher's exact test; $p<0.05$

Table 4. The association between oral health behaviour variables with selected factors (nationality and gender)

\begin{tabular}{|c|c|c|c|}
\hline Variables & OR & $95 \% \mathrm{Cl}$ & $p$ value \\
\hline \multicolumn{4}{|c|}{ Visiting a dentist once a year or more } \\
\hline Female gender & 2.310 & $1.381-3.865$ & 0.001 \\
\hline Polish students & 3.833 & $2.293-6.408$ & $<0.001$ \\
\hline \multicolumn{4}{|c|}{ Visiting a dentist more than a year ago } \\
\hline Female gender & 1.913 & $1.192-3.070$ & 0.007 \\
\hline Polish students & 1.545 & $0.953-2.506$ & 0.078 \\
\hline \multicolumn{4}{|c|}{ Brushing teeth at least once a day } \\
\hline Female gender & 3.759 & $1.567-9.017$ & 0.003 \\
\hline Polish students & 0.530 & $0.212-1.326$ & 0.175 \\
\hline \multicolumn{4}{|c|}{ Brushing teeth 3 minutes or more } \\
\hline Female gender & 0.867 & $0.545-1.379$ & 0.547 \\
\hline Polish students & 2.435 & $1.526-3.885$ & $<0.001$ \\
\hline \multicolumn{4}{|c|}{ Use of electrical toothbrush } \\
\hline Female gender & 0.840 & $0.505-1.400$ & 0.504 \\
\hline Polish students & 1.098 & $0.644-1.874$ & 0.731 \\
\hline \multicolumn{4}{|c|}{ Eating snacks at most occasionally } \\
\hline Female gender & 1.225 & $0.792-1.894$ & 0.361 \\
\hline Polish students & 0.672 & $0.426-1.059$ & 0.087 \\
\hline \multicolumn{4}{|c|}{ Eating sweets less than daily } \\
\hline Female gender & 1.017 & $0.651-1.589$ & 0.941 \\
\hline Polish students & 0.446 & $0.283-0.703$ & 0.001 \\
\hline \multicolumn{4}{|c|}{ Very good opinion of own teeth } \\
\hline Female gender & 0.846 & $0.508-1.408$ & 0.519 \\
\hline Polish students & 0.565 & $0.338-0.945$ & 0.30 \\
\hline
\end{tabular}


Continued from the previous page

Table 4. The association between oral health behaviour variables with selected factors (nationality and gender)

\begin{tabular}{|c|c|c|c|}
\hline Variables & OR & $95 \% \mathrm{Cl}$ & $p$ value \\
\hline \multicolumn{4}{|c|}{ Taking fluoride tablets as a child } \\
\hline Female gender & 1.053 & $0.597-1.858$ & 0.858 \\
\hline Polish students & 0.064 & $0.036-0.113$ & $<0.001$ \\
\hline \multicolumn{4}{|c|}{ Paying attention to toothpaste } \\
\hline Female gender & 2.386 & $1.274-4.471$ & 0.007 \\
\hline Polish students & 1.286 & $0.685-2.412$ & 0.434 \\
\hline \multicolumn{4}{|l|}{ Use of dental floss } \\
\hline Female gender & 2.249 & $1.445-3.503$ & $<0.001$ \\
\hline Polish students & 0.724 & $0.456-1.151$ & 0.172 \\
\hline \multicolumn{4}{|l|}{ Use of mouthwash } \\
\hline Female gender & 1.568 & $0.999-2.460$ & 0.051 \\
\hline Polish students & 1.003 & $0.628-1.604$ & 0.988 \\
\hline \multicolumn{4}{|c|}{ Use of sugar free gum } \\
\hline Female gender & 1.417 & $0.917-2.190$ & 0.116 \\
\hline Polish students & 1.723 & $1.098-2.704$ & 0.018 \\
\hline \multicolumn{4}{|c|}{ Use of dental sticks } \\
\hline Female gender & 0.651 & $0.327-1.139$ & 0.133 \\
\hline Polish students & 1.243 & $0.685-2.254$ & 0.474 \\
\hline \multicolumn{4}{|c|}{ Use of tongue scrapes } \\
\hline Female gender & 1.680 & $0.901-3.133$ & 0.103 \\
\hline Polish students & 0.618 & $0.338-1.131$ & 0.119 \\
\hline \multicolumn{4}{|c|}{ Use of interdental brushes } \\
\hline Female gender & 5.341 & $1.199-23.782$ & 0.028 \\
\hline Polish students & 3.679 & $0.822-16.461$ & 0.088 \\
\hline \multicolumn{4}{|l|}{ Cigarettes smoking } \\
\hline Female gender & 0.613 & $0.373-1.007$ & 0.053 \\
\hline Polish students & 2.340 & $1.336-4.098$ & 0.003 \\
\hline
\end{tabular}

Table 5. The distribution of responses to four statements according to the academic year

\begin{tabular}{|l|c|c|c|c|c|c|c|c|}
\hline & $\begin{array}{c}\text { Dental visits once a } \\
\text { year or more } \\
\mathbf{n}(\%)\end{array}$ & $\mathbf{p}$ & $\begin{array}{c}\text { Twice daily tooth } \\
\text { brushing or more } \\
\mathbf{n}(\%)\end{array}$ & $\mathbf{p}$ & $\begin{array}{c}\text { Use of dental floss } \\
\mathbf{n}(\%)\end{array}$ & $\mathbf{p}$ & $\begin{array}{c}\text { Eating sweets at } \\
\text { least once a day } \\
\mathbf{n}(\%)\end{array}$ & $\mathbf{p}$ \\
\hline Total & $260(100.0)$ & $\mathrm{ns}$ & $324(100.0)$ & $\mathrm{ns}$ & $197(100.0)$ & $\mathrm{ns}$ & $142(100.0)$ & $\mathrm{ns}$ \\
\hline 4th year & $64(24.6)$ & $\mathrm{ns}$ & $81(25.0)$ & $\mathrm{ns}$ & $48(24.3)$ & $\mathrm{ns}$ & $32(22.6)$ & $\mathrm{ns}$ \\
\hline 5th year & $98(37.7)$ & $\mathrm{ns}$ & $117(36.1)$ & $\mathrm{ns}$ & $70(35.6)$ & $\mathrm{ns}$ & $50(35.2)$ & $\mathrm{ns}$ \\
\hline 6th year & $98(37.7)$ & $\mathrm{ns}$ & $126(38.9)$ & $\mathrm{ns}$ & $79(40.1)$ & $\mathrm{ns}$ & $60(42.2)$ & $\mathrm{ns}$ \\
\hline
\end{tabular}

Fisher's exact test; $p<0.05$

ing cessation counselling for medical students would probably reduce the number of smokers and would be effective in preventing smoking initiation. This has been already established at the university in the Czech Republic (27). Nonetheless, further measures are necessary to discourage smoking among Polish medical students.

The results concerning fluoride tablets recommendation revealed that Nordic and Polish policies are different. Nordic students declared having used fluoride tablets almost as often as Polish students declared not having used them. Obviously the use of fluorides contributes substantially to the reduction of dental caries and can be administered in the form of toothpaste, mouthwash, gels or by professionals in the form of varnishes. But there may be a higher risk of dental fluorosis if fluorides are administered in the form of tablets. In literature we can find as many publications for as against such tablets $(5,28,29)$. In Poland, fluoride tablets are not recommended as a regular part of childhood dental regime. In contrast, according to Haugejorden 
and Birkeland (30), in Nordic countries lower caries experience has been attributed to increased sales of fluoride supplements.

Assuming that health awareness level and access to dental information is the same for the entire study group through the ubiquity of the Internet, there must be another explanation why the two nationalities are diverse in dental health behaviour. Scandinavian students who study in Poland have changed their environment but their oral hygiene behaviour has remained unchanged. The fact that they presented very consistent dental health behaviour is meaningful. The situation is different in the case of their Polish counterparts, whose questionnaire results are quite diverse in many aspects. Such outcomes are not merely a coincidence because in another study conducted by Stenberg et al. (31), Swedish respondents up to 25 years of age also gave very consistent responses regarding dental health, and that was over 10 years ago. The author explained that in Sweden dental public health service pays great attention to establishing good dental behaviour among patients. They are educated and taught to attend dental examination once or twice a year. Additionally, the public dental system covers all procedures free of charge for patients up to the age of 18 . So, throughout their life, from the very beginning, young people are given proper oral hygiene motivation and instructions. They are provided with regular care regarding dental caries prevention from dental practitioners, which allows them to adopt good oral hygiene habits (31-34). As one significant factor, we may take under consideration the dental health system. In Poland, in the private sector all procedures are paid and access is easy, while in the dental public service which is available for all insured people, some procedures are free of charge and limited, furthermore, there are waiting lists. Moreover, responsibility for the dental health of a child is placed mostly on the parents who are to organize treatment for the children and there are differences in school-based oral health promotion programmes among Scandinavian countries and Poland. We assume that the Polish public dental system had not influenced the dental behaviour of the Polish students enough considering their diverse answers. This was in opposition to the almost homogenous outcomes of the Scandinavian students. According to Astrom (32), maintaining good oral health related behaviour between ages 15 and 23 is a major assumption of health promotion that can be carried through life. The public dental service in Nordic countries (Finland, Denmark, Norway and Sweden) is considered unique in Europe. According to Widstrom et al. $(35,36)$, it guaranties equal access to oral health service for all members of the population on equal terms. All children are automatically summoned to dental examinations and necessary treatment.

As future medical leaders, the oral attitudes of medical students not only affect their own oral health behaviour but may also influence the health behaviour of their patients. One limitation of our study was the participation rate of Polish students. We cannot be sure about whether they are representative of other medical students in Poland, thus further surveys are needed. We assume that the very high participation rate of the Scandinavians was due to the fact that they represent a rather small group of students (approximately 50 in each year, so they know one another well), compared to 180 Polish students in each year. Another limitation was the use of a self-assessment questionnaire instead of ready questionnaire, which would be much easier to compare with other results. In self reported surveys there is a possibility of biased answers towards the expected behaviour (35). Moreover, we did not change the questions that demonstrated lower reproducibility because the possible answers might differ in different time intervals. Further studies estimating if 15 hours of obligatory dental lectures have any influence on medical students' oral health behaviour should be conducted in the 4 th year.

\section{CONCLUSIONS}

Scandinavian and Polish medical students presented satisfactory oral health behaviour though marked differences were observed. Nordic students' dental health behaviour was very consistent even after the change in their social environment. Polish males and females were the most diverse groups regarding oral behaviour. Polish females took greater care of their teeth than Polish males and the majority of their Scandinavian peers. Nonetheless, a better prognosis for maintaining good oral health has been established in the Scandinavian group of students compared to their Polish counterparts.

\section{Acknowledgements}

We would like to thank the Scandinavian and Polish students for their cooperation in the completion of the survey. The study was founded by grant 113-09648L.

\section{Conflict of Interests}

None declared

\section{REFERENCES}

1. Petersen PE, Bourgeois D, Ogawa H, Estupinan-Day S, Ndiaye C. The global burden of oral diseases and risks to oral health. Bull World Health Organ. 2005 Sep;83(9):661-9.

2. Petersen PE. Sociobehavioural risk factors in dental caries - international perspectives. Community Dent Oral Epidemiol. 2005 Aug;33(4):274-9.

3. Ramirez JH, Arce R, Contreras A. Why must physicians know about oral diseases? Teach Learn Med. 2010 Apr;22(2):148-55.

4. Hugoson A, Koch G. Thirty year trends in the prevalence and distribution of dental caries in Swedish adults (1973-2003). Swed Dent J. 2008;32(2):57-67.

5. Eckersten C, Pylvänen L, Schröder U, Twetman S, Wennhall I, Matsson L. Prevalence of dental fluorosis in children taking part in an oral health programme including fluoride tablet supplements from the age of 2 years. Int J Paediatr Dent. 2010 Sep 1;20(5):347-52.

6. Małkiewicz E, Wierzbicka M, Szatko F, Strużycka I, Ganowicz M, Zawadziński M. Monitoring of oral health. Oral health status and its determinants, in conjunction with prevention of dental caries in children aged 6-12, and adults aged 35-44, Poland; 2010. Warszawa: Ministerstwo Zdrowia; 2010. (In Polish.)

7. Benzian H, Hobdell M, Holmgren C, Yee R, Monse B, Barnard JT, et al. Political priority of global oral health: an analysis of reasons for international neglect. Int Dent J. 2011 Jun;61(3):124-30.

8. Loster BW, Likeman PR. Teaching of dentistry to medical students in English at the Jagiellonian University, Krakow, Poland. Eur J Dent Educ. 2012 Feb;16(1):e1-2.

9. Hodačová L, Šmejkalová J, Čermáková E, Slezák R, Jacob V, Hlaváčková E. Oral health-related quality of life in Czech population. Cent Eur J Public Health. 2010 Jun;18(2):76-80.

10. Rodakowska E, Mierzyńska K, Bagińska J, Jamiołkowski J. Quality of life measured by OHIP-14 and GOHAI in elderly people from Bialystok, north-east Poland. BMC Oral Health. 2014 Aug 20;14:106.

11. Sharda AJ, Shetty S. A comparative study of oral health knowledge, attitude and behavior of first and final year dental students of Udaipur city, Rajasthan, India. Int J Dent Hyg. 2008 Nov;6(4):347-53. 
12. Niiranen T, Widström E, Niskanen T. Oral Health Care Reform in Finland - aiming to reduce inequity in care provision. BMC Oral Health. 2008 Jan 28:8:3.

13. Ordell S, Söderfeldt B. Management structures and beliefs in a professional organisation. An example from Swedish Public Dental Health Services. Swed Dent J. 2010;34(3):167-76.

14. Kawamura M, Ikeda-Nakaoka Y, Sasahara H. An assessment of oral selfcare level among Japanese dental hygiene students and general nursing students using the Hiroshima University--Dental Behavioural Inventory (HU-DBI): surveys in 1990/1999. Eur J Dent Educ. 2000 May; 4(2): 82-8.

15. Ohshima M, Zhu L, Yamaguchi Y, Kikuchi M, Nakajima I, Langham $\mathrm{CS}$, et al. Comparison of periodontal health status and oral health behavior between Japanese and Chinese dental students. J Oral Sci. 2009 Jun;51(2):275-81

16. Kumar S, Kriplani D, Shah V, Tadakamadla J, Tibdewal H, Duraiswamy $\mathrm{P}$, et al. Oral health attitudes and behaviour as predisposing factor for dental caries experience among health professional and other professional college students of India. Oral Health Prev Dent. 2010;8(2):195-202.

17. Paulander J, Axelsson P, Lindhe J. Association between level of education and oral health status in 35-, 50-, 65- and 75-year-olds. J Clin Periodontol. 2003 Aug;30(8):697-704.

18. Baginska J, Rodakowska E, Milewski R, Kierklo A. Dental caries in primary and permanent molars in 7-8-year-old schoolchildren evaluated with Caries Assessment Spectrum and Treatment (CAST) index. BMC Oral Health. 2014 Jun 21;14:74.

19. Khami MR, Virtanen JI, Jafarian M, Murtomaa H. Oral health behaviour and its determinants amongst Iranian dental students. Eur J Dent Educ. 2007 Feb;11(1):42-7.

20. Kirtiloğlu T, Yavuz US. An assessment of oral self-care in the student population of a Turkish university. Public Health. 2006 Oct;120(10):953-7.

21. Rimondini L, Zolfanelli B, Bernardi F, Bez C. Self-preventive oral behavior in an Italian university student population. J Clin Periodontol. 2001 Mar;28(3):207-11.

22. Al-Ansari J, Honkala E, Honkala S. Oral health knowledge and behavior among male health sciences college students in Kuwait. BMC Oral Health. 2003 May 7;3(1):2.

23. World Health Organization [Internet]. Geneva: WHO; c2016 [cited 2016 Feb 1]. Tobacco Control Country Profiles. Available from: http://www. who.int/tobacco/surveillance/policy/country_profile/en/index.html

24. Vellappally S, Jacob V, Smejkalová J, Shriharsha P, Kumar V, Fiala Z. Tobacco habits and oral health status in selected Indian population. Cent Eur J Public Health. 2008 Jun;16(2):77-84.
25. Inandi T, Caman OK, Aydin N, Onal AE, Kaypmaz A, Turhan E, et al. Global Health Professions Student Survey - Turkey: second-hand smoke exposure and opinions of medical students on anti-tobacco law. Cent Eur J Public Health. 2013 Sep;21(3):134-9.

26. Vanderhoek AJ, Hammal F, Chappell A, Wild TC, Raupach T, Finegan BA. Future physicians and tobacco: an online survey of the habits, beliefs and knowledge base of medical students at a Canadian University. Tob Induc Dis. 2013 Apr 4;11(1):9.

27. Schneidrová D, Kopřivová Herotová TK, Šustková M. Smoking Prevention Activities at the Third Faculty of Medicine of Charles University in Prague, Czech Republic. Cent Eur J Public Health. 2012 Mar;20(1):67.

28. Banting DW. International fluoride supplement recommendations. Community Dent Oral Epidemiol. 1999 Feb;27(1):57-61.

29. Meyer-Lueckel H, Grundmann E, Stang A. Effects of fluoride tablets on caries and fluorosis occurrence among 6- to 9-year olds using fluoridated salt. Community Dent Oral Epidemiol. 2010 Aug;38(4):315-23.

30. Haugejorden O, Birkeland JM. Analysis of the ups and downs of caries experience among Norwegian children aged five years between 1997 and 2003. Acta Odontol Scand. 2005 Apr;63(2):115-22.

31. Stenberg P, Håkansson J, Åkerman S. Attitudes to dental health and care among 20 to 25-year-old Swedes: results from a questionnaire. Acta Odontol Scand. 2000 Jun;58(3):102-6.

32. Astrøm AN. Stability of oral health-related behaviour in a Norwegian cohort between the ages of 15 and 23 years. Community Dent Oral Epidemiol. 2004 Oct;32(5):354-62.

33. Jensen O, Gabre P, Sköld UM, Birkhed D. Fluoride toothpaste and toothbrushing; knowledge, attitudes and behavior among Swedish adolescents and adults. Swed Dent J. 2011;35(4):203-13

34. Albertsson KW, van Dijken JW. Awareness of toothbrushing and dentifrice habits in regularly dental care receiving adults. Swed Dent J. 2010;34(2):71-8.

35. Widström E, Eaton KA. Oral healthcare systems in the extended European union. Oral Health Prev Dent. 2004;2(3):155-94.

36. Widström E, Ekman A, Aandahl LS, Pedersen MM, Agustsdottir H, Eaton KA. Developments in oral health policy in the Nordic countries since 1990. Oral Health Prev Dent. 2005;3(4):225-35.

Received August 30, 2014 Accepted in revised form September 23, 2015 